\title{
Hydrogenation effect on the crystallization of alloys leading to the development of nanostructured states
}

\author{
N.E. SKRYABINA ${ }^{1,2}$, D. FRUCHART ${ }^{2}$, A.V. SHELYAKOV ${ }^{3}$ \\ ${ }^{1}$ Perm State University, 15 Bukireva, 614990, Perm, Russia \\ ${ }^{2}$ Laboratoire de Cristallographie, CNRS, BP 166, 38042 Grenoble cedex 9, France \\ ${ }^{3}$ Moscow Engineering Physics Institute, 31 Kashirskoe Sh., 115409 Moscow, Russia \\ *Corresponding author. E-mail: magicflight@permonline.ru
}

Received February 15, 2008; accepted February 17, 2008; available on-line March 31, 2008

Two original effects were evidenced upon hydrogenation of $\mathrm{Ti}_{50} \mathrm{Ni}_{25} \mathrm{Cu}_{25}$ alloys. Saturation of amorphous state $\mathrm{Ti}_{50} \mathrm{Ni}_{25} \mathrm{Cu}_{25}$ alloys with hydrogen induces the formation of a nanocrystalline structure after annealing. The main characteristic of the nanocrystalline state is the appearance of two steps of crystallization. When the temperature of the first step is reached, crystallization leads to precipitation of B2-type nucleation centers in an amorphous matrix. The well established martensite phase transformation, which occurs when cooling a sample down to the first step crystallization, is fully suppressed in the case of the hydrogen-treated materials.

Amorphous materials / Phase transformation / X-ray diffraction

\section{Introduction}

A wide panel of methods can be used to develop nanocrystalline structures, depending on the material to be processed. With this purpose, we considered TiNi-based alloys. TiNi type compounds are of interest due to their specific properties, since many of them exhibit high-level shape memory properties, as well as pronounced super-elastic characteristics. It is known that for this type of compounds, a nanocrystalline state can be induced by using two specific routes. The first process consists in meltquenching amorphous ribbons, and then to form a nanocrystalline state of B2-type structure, immediately after inducing a primary crystallization treatment, by annealing in the temperature range 400$450^{\circ} \mathrm{C}$. During subsequent cooling of the sample to room temperature, the martensitic transformation B2 $\rightarrow$ B19 takes place. The second process consists in applying a particular quenching rate during the meltspinning operation. Ideally, the quenching rate should be approximately $510^{5} \mathrm{~K} / \mathrm{s}$. In both cases, the resulting ribbons exhibit structures of the B19martensite type at room temperature.

It has been shown earlier that by applying an hydrogenation procedure, a new type of crystalline structure can be developed at room temperature in Ti-Ni alloys [1,2]. In this work we will not discuss the potential formation of a new hydride phase, but we will examine the results of a specific hydrogenation process able to create microstructure re-arrangements involving all three metal elements of the formula.

\section{Experimental}

Amorphous ribbons with the composition $\mathrm{Ti}_{50} \mathrm{Ni}_{25} \mathrm{Cu}_{25}$ were prepared using the melt spinning technique. Nanocrystalline state samples (standard) were obtained by heating the ribbons up to $450^{\circ} \mathrm{C}$ under a pure argon atmosphere. The crystallization process was investigated using a differential scanning calorimeter Perkin-Elmer DSC-7. For this experiment, the heating rate was $5 \mathrm{~K} / \mathrm{min}$. X-ray diffraction patterns were recorded in the Bragg-Brentano mode using a Philips PW1720 diffractometer working at $\lambda_{\text {CuK } \alpha}$ and equipped with a backscattering graphite monochromator. The patterns were collected at room temperature with a step $2 \theta=0.05^{\circ}$. Hydrogen was loaded at room temperature using a $\mathrm{H}_{2} \mathrm{SO}_{4}$ based electrolyte, at a $50 \mathrm{~A} / \mathrm{m}^{2}$ current density. The sample was the cathode and the anode was realized with a platinum wire.

\section{Results and discussion}

The phase diagram of the pseudo binary $\mathrm{Ti}_{50} \mathrm{Ni}_{25} \mathrm{Cu}_{25}$ alloy under investigation can be found in reference [3]. A nanocrystalline structure was developed by 
application of a specific annealing treatment to the amorphous state ribbon. A thermoelastic B2 $\rightarrow$ B19 martensite-type transformation is known to take place at room temperature after having cooled down the sample. Then, the crystallized $\mathrm{Ti}_{50} \mathrm{Ni}_{25} \mathrm{Cu}_{25}$ material exhibits the orthorhombic B19 martensite structure type, as shown in Fig. 1, Pattern 2.

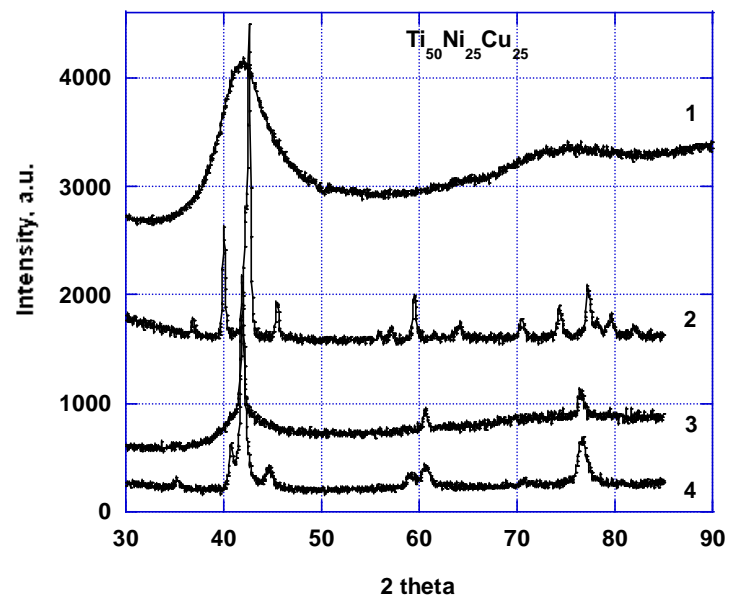

Fig. 1 X-ray diffraction patterns of $\mathrm{Ti}_{50} \mathrm{Ni}_{25} \mathrm{Cu}_{25}$ alloys: 1 - initial amorphous state, 2 - after annealing at $450^{\circ} \mathrm{C}, 3$ - after hydrogenation and annealing at $450^{\circ} \mathrm{C}$, 4 - after hydrogenation and two cycles of annealing at 450 and $520^{\circ} \mathrm{C}$, respectively.

We found that hydrogen loading of amorphous $\mathrm{Ti}_{50} \mathrm{Ni}_{25} \mathrm{Cu}_{25}$ alloys generally results in a shift of the crystallization step to higher temperatures, as shown in Fig. 2.

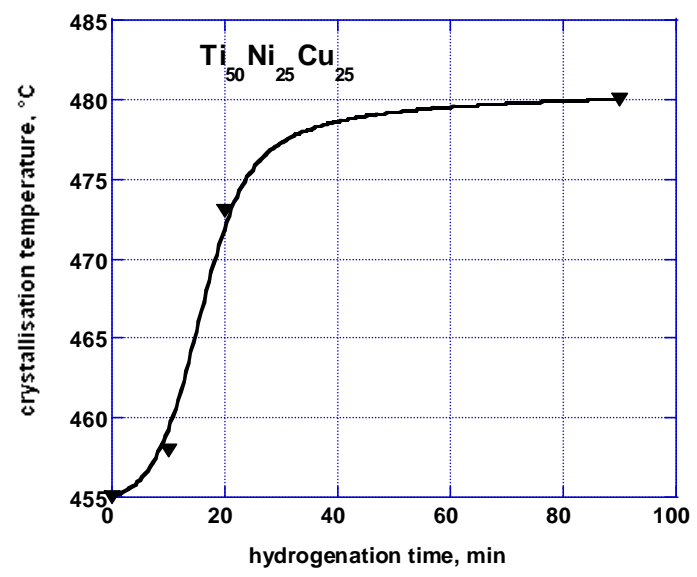

Fig. 2 Full crystallization temperature vs. hydrogenation time for $\mathrm{Ti}_{50} \mathrm{Ni}_{25} \mathrm{Cu}_{25}$ alloys.

A typical duration of the hydrogenation process of up to 90 minutes led to the appearance of new features of the crystallisation process, as determined from DSC analysis. Firstly, two steps of crystallisation were found and secondly, the temperature of full crystallisation was shifted $25^{\circ} \mathrm{C}$ higher, with respect to the standard sample (Fig. 3, Trace 1). The first one of the two crystallisation steps corresponds to a firstlevel nucleation process where nano-sized grains are formed within a typical nanocrystalline, but not homogeneous structure. In order to realize a pertinent $\mathrm{X}$-ray analysis, the sample was first heated to $450^{\circ} \mathrm{C}$ and then cooled down to room temperature. Careful examination of the resulting X-ray diffraction pattern revealed that after the first crystallization step, the structure of the sample corresponds to a mixture of two components. From Fig. 1, Pattern 3, it can be seen that the first component has the $b c c$ B2 structure type, whereas the second one remains amorphous, as evidenced from the background modulation. A subsequently performed DSC analysis confirmed fairly well this result. At the second stage, the hydrogen-treated sample was investigated in the same range of temperature as the hydrogen-free sample. Contrary to what was observed during the former experiment, for the hydrogen-treated sample the martensite type of transformation appeared to be completely suppressed. Nucleation of nano-grains in a non-crystallized matrix, resulting from the first crystallization step, has made impossible the expected B2 $\rightarrow$ B19 transformation. The blocking phenomenon must be related to the specific reduced size of the grains, and occurs in particular for those nano-crystals that are fully embedded in the amorphous matrix. On the contrary, the B2 $\rightarrow$ B19 structural phase transformation should develop from fractions of nanocrystals of larger size. It is worth recalling that the specific size effect enabling or not a martensite phase transformation has been thoroughly described in a review paper [4].

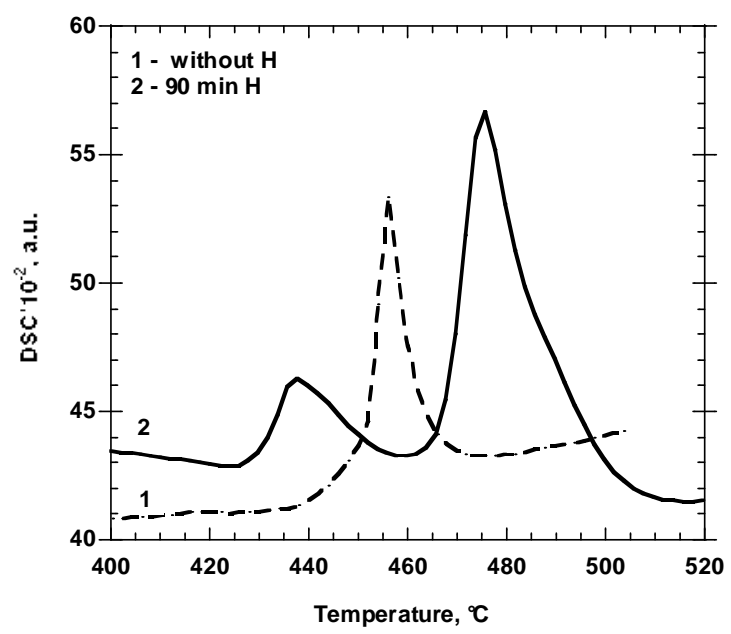

Fig. 3 DSC analysis of $\mathrm{Ti}_{50} \mathrm{Ni}_{25} \mathrm{Cu}_{25}$ samples: 1 - hydrogen free (fresh sample), 2 - hydrogen treated for $90 \mathrm{~min}$. before annealing.

Evidently, after full crystallization the structure of the hydrogenated material differs from that of the hydrogen-free sample. For the hydrogen-treated ribbons, the X-ray diffraction analysis reveals two different phases (Fig. 1, Pattern 4). The B2-type component, having crystallized at first, remains the 
dominating one, however the B19'-type monoclinic type of structure has developed in parallel. Such a quite original situation is markedly different from that resulting from the crystallization of a fresh (non hydrogenated) ribbon, where a unique phase of the B19 structure type is formed. The formation of a B19' component, instead of the expected B19 one, at the second crystallization step of the hydrogen-treated material implies a significant redistribution of the metal atoms. Obviously, this selective effect results from both the hydrogenation and the first-step crystallization process. However, one can consider that during the final nucleation and growth process, the B19' structure, which has lower symmetry than the B19 structure, is more inclined towards accommodating larger levels of stress like those existing in hydrogenated samples. Finally, it should be noted that the hydrogenation treatment induces a more stable state, which is fully retained even after several heating cycles up to the crystallization temperature.

\section{Conclusion}

Hydrogen-processing of $\mathrm{Ti}_{50} \mathrm{Ni}_{25} \mathrm{Cu}_{25}$ ribbons has revealed new trends in nanostructured materials. It was shown that hydrogen acts as a structure-forming agent, making it possible to stabilize a specific nanocrystalline structure. When applied to an amorphous state, the hydrogen-structuration process operates as a driving force, similarly to specific thermal treatments. After hydrogenation and the corresponding first crystallization process, the soformed nanostructure remains stable for long times. The ability of hydrogen to increase the stability of nanocrystalline type structures is probably not restricted to metal systems such as $\mathrm{Ti}_{50} \mathrm{Ni}_{25} \mathrm{Cu}_{25}$ alloys.

\section{Acknowledgements}

We gratefully thank both the Russian Foundation for Basic Research, Grant No 03-02-16561a and the ECONET Project No 08133RA of the French Foreign Office, for financial support.

\section{References}

[1] M.H. Shorshorov, I.A. Stepanov, U.M. Flomenbli, V.V. Travkin, Dokl. Akad. Nauk SSSR 283(2) (1985) 370-373.

[2] N.E. Skryabina, D. Fruchart, L. Spivak, J. Phys. IV (France) 115 (2004) 215-221.

[3] F.J.J. Van Loo, G.F. Bastin, A.J.H. Leenen, J. Less-Common Met. 57 (1978) 111-117.

[4] A.M. Glezer, E.N. Blinova, V.A. Pozdnyakov, A.V. Shelyakov, J. Nanoparticle Res. 5 (2003) 551-560.

Proceeding of the IX International Conference on Crystal Chemistry of Intermetallic Compounds, Lviv, September 20-24, 2005. 